\title{
EDUCACIÓN MÉDICA Y PANDEMIA: VALORACIÓN DE LOS CAMBIOS IMPUESTOS POR LAS RESTRICCIONES DE LA PANDEMIA EN LA DOCENCIA-APRENDIZAJE DE LA ANATOMÍA SEGÚN LOS ESTUDIANTES DE PRIMER AÑ̃ DE MEDICINA
}

\author{
Susana N. Biasutto, Luisina Cuadrado, Juan P. Novillo, María de la P. \\ Postacchini, Valentina Sánchez Barneix, M. Milena Viccini, Matías A. Perea, \\ Nicolás Longoni
}

\author{
Cátedra de Anatomía Normal, Facultad de Ciencias Médicas, Universidad Nacional de \\ Córdoba, Córdoba, Argentina
}

\begin{abstract}
RESUMEN
Afrontar las restricciones de la pandemia antes siquiera de comenzar el ciclo lectivo fue un gran desafío para los estudiantes de primer año de Medicina. Repentinamente las universidades se cerraron y se debió reestructurar toda la actividad académica. El presente trabajo tiene por objetivo valorar la opinión de los alumnos de Anatomía que debieron cursar en 2020 sin actividades presenciales y quienes cursan en el presente año con escasa asistencia, exclusivamente para la parte práctica. Se realizó una encuesta en línea para que evaluaran los factores que incidieron en su formación anatómica durante este periodo de condiciones tan particulares. Determinamos 3 grupos: los alumnos que cursaron en 2020, los que cursan actualmente y los que comenzaron en 2020 y vuelven a cursar en 2021. La mayoría no había tenido experiencia de estudio virtual previamente. La valoración de las clases fue media y el principal problema fue la falta de acceso a los cuerpos y el material cadavérico para el estudio de la parte practica, seguido por la ansiedad, falta de motivación y concentración para el estudio, la falta de interacción con sus compañeros y docentes. La ventaja más destacada fue el ahorro de tiempo y, en menor medida, la reducción de gastos. En conclusión, los estudiantes consideran que la pérdida más importante se basa en la falta de acceso a los cuerpos y material cadavérico, aunque la mayoría tiene expectativas de recuperarlo por diferentes vías. En general consideran que la pandemia tendrá serias consecuencias en su formación médica.
\end{abstract}

Palabras clave: Anatomía, educación médica, pandemia, restricciones

\begin{abstract}
To afford the pandemic restrictions when the universitary year even started was a great challenge for first-year medical students. Suddenly universities were closed and all academic activity had to be reorganized. The objective of this study is to assess the opinion of Anatomy students who developed the course in 2020 without face-to-face activities and those who are doing this year with little attendance, exclusively for the practical part. An online survey was conducted to evaluate the factors that influenced their anatomical formation during a period of such particular conditions. We determined 3 groups: students who studied in 2020, those who are currently studying and those who started in 2020 and return to study in 2021. Most of them had not previously had a virtual study experience. The classes assessment was medium and the main problem was the lack of access to bodies and cadaveric material for the study of the practical aspects, followed by anxiety, lack of motivation and concentration for the study, lack of interaction with classmates and professors. The most outstanding advantage was the saving of time and, to a lesser extent, reduction of costs. In conclusion, students considered the most important loss was the lack of access to corpses and cadaveric material, although most of them had expectations of recovering the experience by different ways. In general, they considered the pandemic will have serious consequences on their medical training.
\end{abstract}

Key words: Anatomy, medical education, pandemic, restrictions

\footnotetext{
* Correspondencia a: Prof. Dr. Susana N. Biasutto subiasutto@gmail.com
}

Recibido: 14 de Junio, 2021. Revisado: 26 de Junio, 2021. Aceptado: 10 de Julio, 2021. 


\section{INTRODUCCIÓN}

La enseñanza-aprendizaje de la Anatomía Humana ha pasado por diferentes cambios en épocas recientes. $A$ fines del siglo $X X$ e inicios del XXI se difundió la idea de que los advances tecnológicos podrían reemplazar el uso del material cadavérico. Aunque los recursos digitales para la enseñanza han aportado y facilitado el estudio, no han podido reemplazar a los cuerpos humanos en su posibilidad de identificar texturas, elasticidad, tridimensionalidad, relaciones, variaciones anatómicas, etc., habiendo sido reconocidos como irremplazables en la actualidad (Biasutto et al, 2006; Azer and Eizenberg, 2007; Cahill and Ettarh, 2008; Sugand et al, 2010; Dereje, 2014; NarvaezHernandez and Murillo-Rabago, 2014; ArráezAybar et al, 2014).

La pandemia por COVID-19, que se iniciara en China a fines de 2019 y continua aún en 2021, ha impuesto un nuevo desafío para el mundo, en el que las Ciencias de la Salud se ven muy comprometidas tanto en la práctica professional, la investigación y la formación de recursos humanos. Los gobiernos impusieron restrictciones para evitar el contacto próximo entre las personas, y en este marco, la gran mayoría de las actividades comenzaron a desarrollarse a distancia, apelando al uso de la tecnología (Bond y Franchi, 2020; Dost et al., 2020; Longhurst et al., 2020; Pather et al., 2020; Puljak et al., 2020; Singal et al, 2020; Banovac et al., 2021; Deutschman et al., 2021; Pawlina, 2021; Shahrvini et al., 2021; Singal et al, 2021; Suarez-Escudero, et al., 2021)

Las restricciones por la pandemia de COVID-19 en Argentina comenzaron el 20 de marzo del 2020, fecha similar a la mayoría de los países del mundo, solo que en las universidades argentinas aún no habían comenzado los cursos regulares del ciclo lectivo correspondiente a ese año. Prevalecían las medidas sanitarias y la educación fue relegada. Las universidades se cerraron para los estudiantes, aunque de inmediato se organizaron actividades virtuales para suplir los requerimientos de la formación académica. Los alumnos de primer año de Medicina habían apenas ingresado a la Universidad y no llegaron a conocer siquiera el aula o la Sala de Disección en la Cátedra de Anatomía. El acceso a pacientes y cadáveres se vio restringido por las medidas de bioseguriadad. Las instituciones, los docentes y los alumnos no estaban preparados para afrontar el desafío repentino que se presentaba. Todas las partes intervinientes hicieron su mejor esfuerzo y progresivamente se fueron adaptando y mejorando. Cuál fue el impacto y las repercusiones de ese proceso, que aún continúa, para los estudiantes de Anatomía?. El objetivo de este estudio es determinar la valoración que ellos realizan de los factores que incidieron en su formación anatómica durante este periodo de condiciones tan particulares.

\section{MATERIAL Y MÉTODOS}

Se realizó un estudio transversal cualitativo y relacional, basado en una encuesta anónima y voluntaria, con respuestas por selección múltiple, semi-estructuradas y otras con escala de valoración de 1 a 5 (tipo Likert).

La encuesta se confeccionó para realizarse en línea y se distribuyó entre todos los estudiantes que cursaron Anatomía Normal en 2020 y 2021 , considerando que debido a la pandemia en 2020 los estudiantes no tuvieron actividades presenciales y en 2021 los trabajos prácticos en la Sala de Disección de la Cátedra han sido escasos hasta el momento y en grupos muy pequeños debido a las medidas restrictivas de distanciamiento. Se tomaron las medidas de resguardo para evitar la duplicación de las respuestas.

Se incluyeron preguntas sobre edad, género, nacionalidad, provincia de origen de los argentinos, año en que cursó Anatomía Normal, dificultades, ventajas y desventajas para el estudio virtual y de la Anatomía en particular, nivel de satisfacción con las actividades organizadas por la Cátedra, tiempo de dedicación al estudio, importancia del aprendizaje práctico con cuerpos, importancia de la Anatomía para la carrera y la incidencia de las restricciones por la pandemia en todo ello. Las preguntas se confeccionaron en función a variables percibidas por los docentes que podrían influir en el proceso de aprendizaje y otras evaluadas en la literatura consultada.

Haber cursado la asignatura en un año diferente a 2020 y 2021 se consideró como único criterio de exclusión.

Los resultados se informan en porcentajes o de acuerdo a una escala de valoración de 1 a 5 , donde 1 es "nada" (no afectó nada, nada satisfecho, ningún problema o no me interesa), 5 es "mucho" (afectó mucho, muy satisfecho, mucho problema o muy interesado/a) y la correspondiente escala intermedia. Consideramos diferencia estadísticamente significativa la $P=<0,05$. Para el análisis de los resultados se aplicó la prueba de Chi cuadrado para evaluar la asociación de variables cualitativas.

El programa estadístico usado fue INFOSTAT. 


\section{RESULTADOS}

Setecientos tres estudiantes respondieron la encuesta y seis se excluyeron por haber cursado la materia en un año previo al 2020.

Dadas las diferencias en las condiciones de cursado, se dividieron las respuestas en 3 grupos:

Grupo I: Alumnos que cursaron Anatomía Normal en el ciclo lectivo 2020, sin ninguna posibilidad de acceder a la presencialidad, sin contacto con material cadavérico real y en circunstancias completamente novedosas que requirieron la implementación de nuevas estrategias y recursos docentes

Grupo II: Alumnos que están cursando la asignatura en el 2021, que tuvieron alguna actividad presencial en grupos muy pequeños y alternándose en el tiempo, por lo cual las actividades fueron exclusivamente prácticas, de corta duración y cada 14 días, donde debe considerarse también que la presencialidad no es obligatoria y así, los alumnos que no están viviendo en Córdoba o aquellos que por elección no quieren exponerse, no concurren al ámbito universitario. Estos alumnos aún tienen expectativa de cierta normalidad durante el año en curso

Grupo III: Alumnos que cursaron en el 2020 y están cursando nuevamente en el presente, por diferentes razones. Es un grupo pequeño, con características particulares y diferentes a los demás.

El grupo I incluyó 221 encuestas (tasa de respuesta del $39,22 \%$ y $31,71 \%$ del total de encuestados) correspondientes a 153 mujeres $(69,23 \%), 67$ varones $(30,32 \%)$ y 1 de otro género $(0,45 \%)$, de $20,40 \pm 2,22$ años de edad (min 18, max 33). El grupo II incluyó 455 encuestas (tasa de respuesta de 59,79\% y $65,28 \%$ del total) provenientes de 334 mujeres (73,41\%) y 121 varones (26,59\%), de 19,59 $\pm 2,54$ años de edad ( $\min 16$ y max 40). Al grupo III correspondieron 21 encuestas $(0,3 \%$ del total) de 17 mujeres $(80,95 \%)$ y 4 varones $(19,05 \%)$, de $20,81 \pm 2,77$ años de edad ( $\min 18, \max 28$ ). Debido a que este grupo es muy pequeño, el análisis estadístico de sus resultados tiene valor relativo y solo debe considerarse en el marco del contexto general y a los fines del aporte particular que pudiera hacer a esta investigación. Los extranjeros representaron un porcentaje no significativo $(3,62 \%-2020,2,42 \%-2021$ y $0 \%$ 2020/21). De los argentinos, los estudiantes no originarios de Córdoba fueron el $61 \%$ en el grupo I, el $60 \%$ en el grupo II y el $48 \%$ en el grupo III.

La familiaridad con el estudio por medios digitales antes de la pandemia fue diferente en cada año $(p=<0,0001)$, con un $26 \%$ en el grupo I, un $45 \%$ en el grupo II y un $10 \%$ en el grupo III (Gráfico 1).

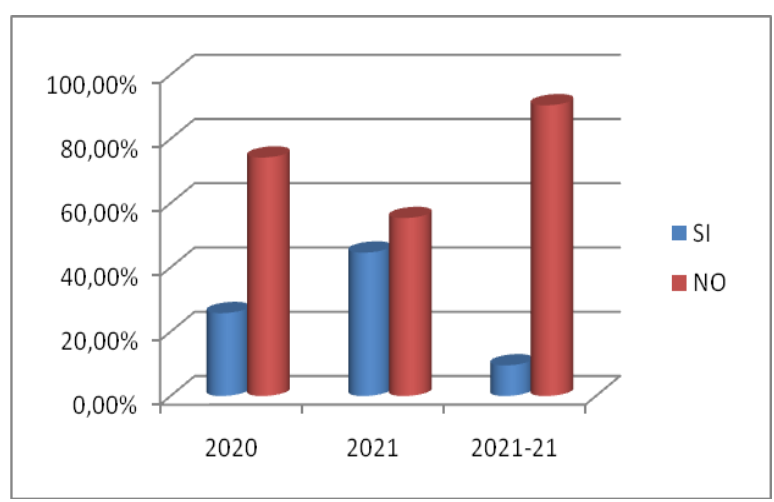

Gráfico 1- Familiaridad con el estudio virtual previamente a la pandemia

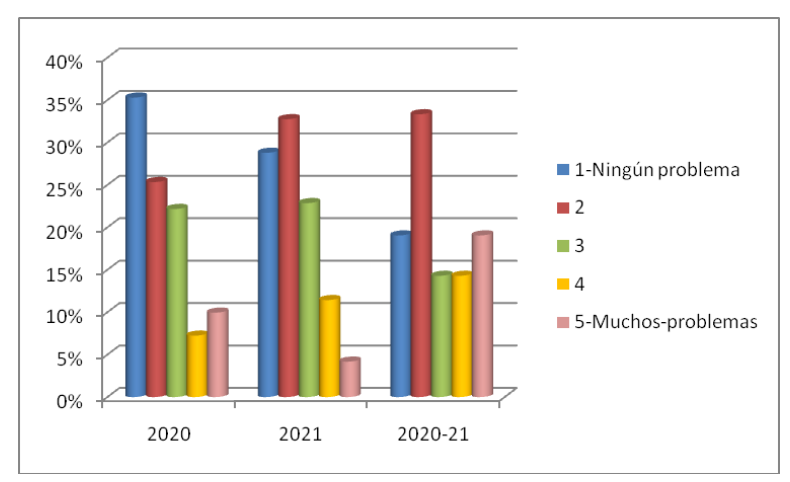

Gráfico 2- Valoración de la interferencia por ruidos molestos en el estudio virtual de la Anatomía

La consideración sobre los problemas técnicos que pudieron tener los alumnos para el estudio virtual de la Anatomía involucró la valoración sobre varios temas (ver Tabla 1). A) La interrupción o dificultosa conexión a internet tuvo una incidencia mayoritariamente media a baja (12-3 de Likert) para los grupos I y II (78\% y $87 \%$, $\mathrm{p}=0,0614)$, y alcanzó altos porcentajes en los valores 3-4-5 de Likert para el grupo III (81\%, $p=0,0059$ ). B) La interferencia por ruidos molestos no evidenció niveles de relevancia aunque con diferencias significativas entre todos los grupos $(p=0,0043)$. La Incidencia mayor se observó en el grupo III (33\% valores 4 y 5 ) y la menor en el grupo II (16\% valores 4 y 5 ). Gráfico 2. C) La dificultad para acceder a las actividades proporcionadas por la Cátedra fue muy baja para todos los grupos. El $57 \%, 67 \%$ y $52 \%$ respectivamente no tuvo ningún problema y si consideramos los valores 3,2 y 1 (dificultad media a ninguna para acceder) los porcentajes ascienden al $96 \%, 98 \%$ y $90 \%(p=0,1263)$. D) Se observó una diferencia significativa entre los 
grupos en relación a la comunicación para realizar consultas $(p=<0,0001)$. Si bien la mayoría de los alumnos refieren no haber tenido problemas $(61 \%$ grupo $1,78 \%$ grupo II y $57 \%$ grupo III), quienes cursaron en 2020 (grupos I y III) evidencian haber tenido más dificultades que quienes cursan en el presente año (grupo II). Gráfico 3.

\begin{tabular}{|c|c|c|c|c|c|c|c|c|c|c|c|c|c|}
\hline & & & \multicolumn{10}{|c|}{ VALORACIÓN TIPO LIKERT } & \multirow[b]{3}{*}{$P=$} \\
\hline & & & \multicolumn{2}{|c|}{$\begin{array}{l}\text { 1- Ningún } \\
\text { problema }\end{array}$} & \multicolumn{2}{|c|}{2} & \multicolumn{2}{|c|}{3} & \multicolumn{2}{|c|}{4} & \multicolumn{2}{|c|}{$\begin{array}{l}\text { 5-Muchos } \\
\text { problema }\end{array}$} & \\
\hline & & & $\mathrm{N}$ & $\%$ & $\mathrm{~N}$ & $\%$ & $\mathrm{~N}$ & $\%$ & $\mathrm{~N}$ & $\%$ & $\mathrm{~N}$ & $\%$ & \\
\hline \multirow[t]{2}{*}{ PROBL. } & \multirow[t]{3}{*}{ Internet } & Grupo 1 & 35 & 15,84 & 77 & 34,84 & 61 & 27,60 & 30 & 13,57 & 18 & 8,14 & \multirow[b]{3}{*}{0,0059} \\
\hline & & Grupo 2 & 91 & 20 & 183 & 40,22 & 120 & 26,37 & 39 & 8,57 & 22 & 4,84 & \\
\hline $\mathbf{T}$ & & Grupo 3 & 2 & 9,52 & 2 & 9,52 & 10 & 47,62 & 5 & 23,81 & 2 & 9,52 & \\
\hline $\mathbf{E}$ & \multirow{3}{*}{$\begin{array}{l}\text { Ruidos } \\
\text { molestos }\end{array}$} & Grupo 1 & 78 & 35,29 & 56 & 25,34 & 49 & 22,17 & 16 & 7,24 & 22 & 9,95 & \multirow[b]{3}{*}{0,0043} \\
\hline C & & Grupo 2 & 131 & 28,79 & 149 & 32,75 & 104 & 22,86 & 52 & 11,43 & 19 & 4,18 & \\
\hline $\mathbf{N}$ & & Grupo 3 & 4 & 19,05 & 7 & 33,33 & 3 & 14,29 & 3 & 14,29 & 4 & 19,05 & \\
\hline I & \multirow{3}{*}{$\begin{array}{c}\text { Acceso } \\
\text { a las } \\
\text { actividades } \\
\end{array}$} & Grupo 1 & 127 & 57,47 & 66 & 29,86 & 19 & 8,60 & 6 & 2,71 & 3 & 1,36 & \multirow[b]{3}{*}{0,1263} \\
\hline C & & Grupo 2 & 307 & 67,47 & 97 & 21,32 & 40 & 8,79 & 7 & 1,54 & 4 & 0,88 & \\
\hline $\mathbf{0}$ & & Grupo 3 & 11 & 52,38 & 5 & 23,81 & 3 & 14,29 & 1 & 4,76 & 1 & 4,76 & \\
\hline \multirow[t]{3}{*}{$\mathbf{S}$} & \multirow{3}{*}{$\begin{array}{c}\text { Acceso } \\
\text { a las } \\
\text { consultas } \\
\end{array}$} & Grupo 1 & 78 & 35,29 & 56 & 25,34 & 49 & 22,17 & 16 & 7,24 & 22 & 9,95 & \multirow[b]{3}{*}{$<0,0001$} \\
\hline & & Grupo 2 & 245 & 53,85 & 112 & 24,62 & 49 & 10,77 & 30 & 6,59 & 19 & 4,18 & \\
\hline & & Grupo 3 & 8 & 38,10 & 4 & 19,05 & 4 & 19,05 & 3 & 14,29 & 2 & 9,52 & \\
\hline
\end{tabular}

Tabla 1- Valoración de los problemas técnicos.

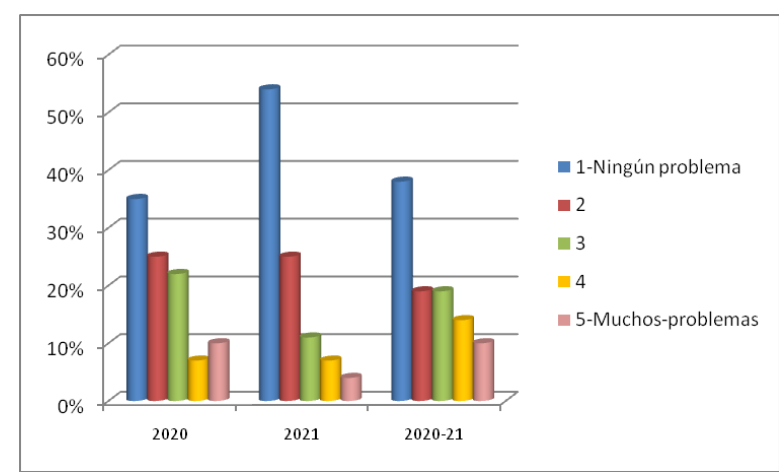

Gráfico 3- Trastornos en la comunicación para realizar consultas

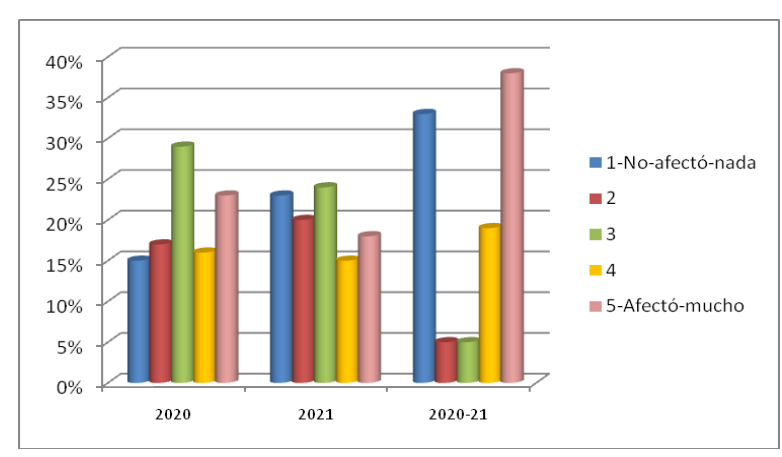

Gráfico 4- Evaluación de la desventaja causada por las distracciones familiares.
Se consultaron varios aspectos que podrían ser considerados como ventajas del estudio virtual: A) El ahorro de tiempo fue importante y constante para los encuestados de todos los grupos, sin evidenciarse diferencias entre ellos $(p=0,9083)$, con valores 3,4 y 5 de Likert en el $87 \%, 85 \%$ y $80 \%$ respectivamente para cada grupo. B) La reducción de gastos no tuvo una repercusión similar al ítem anterior. La distribución fue bastante equilibrada entre los distintos valores (y sin diferencia significativa entre los grupos ( $p=0,1986)$. C y D) En relación a un mejor aprovechamiento del tiempo de estudio y tener mayor comodidad, no hubo diferencias estadísticas entre los grupos en este ítem $(p=0,1181$ y $p=0,5641$ respectivamente) y predominaron los valores medios. E) Se consideró si podría ser una ventaja no enfrentar el contacto directo con el cadáver, a lo cual los alumnos respondieron en forma terminante, con respuestas valor 1 (nada de ventaja) en un $87 \%$, $92 \%$ y $97 \%(p=0,5953)$. F) La flexibilidad de los horarios evidenció niveles de valoración distribuidos de modo bastante uniforme y con bastante similitud entre los grupos $(p=0,3902)$. G) Los alumnos también rechazaron que aprender a su propio ritmo pudiera ser una ventaja (valores $1-2-3$ de Likert de $80 \%$, $85 \%$ y 
$100 \%$ en los grupos I, II y III respectivamente) ( $p=0,1777)$. Consultar resultados en la Tabla 2 .

\begin{tabular}{|c|c|c|c|c|c|c|c|c|c|c|c|c|c|}
\hline & & & \multicolumn{10}{|c|}{ VALORACIÓN TIPO LIKERT } & \multirow[b]{3}{*}{$P=$} \\
\hline & & & \multicolumn{2}{|c|}{ 1- Nada } & \multicolumn{2}{|c|}{2} & \multicolumn{2}{|c|}{3} & \multicolumn{2}{|c|}{4} & \multicolumn{2}{|c|}{ 5-Mucho } & \\
\hline & & & $\mathrm{N}$ & $\%$ & $\mathrm{~N}$ & $\%$ & $\mathrm{~N}$ & $\%$ & $\mathrm{~N}$ & $\%$ & $\mathrm{~N}$ & $\%$ & \\
\hline & \multirow{3}{*}{$\begin{array}{c}\text { Ahorro de } \\
\text { tiempo }\end{array}$} & Grupo 1 & 10 & 4,52 & 18 & 8,14 & 43 & 19,46 & 36 & 16,29 & 114 & 51,58 & \multirow[b]{3}{*}{0,9083} \\
\hline & & Grupo 2 & 21 & 4,62 & 46 & 10,11 & 77 & 16,92 & 69 & 15,16 & 242 & 53,19 & \\
\hline & & Grupo 3 & 2 & 9,52 & 2 & 9,52 & 5 & 23,81 & 2 & 9,52 & 10 & 47,62 & \\
\hline & \multirow{3}{*}{$\begin{array}{l}\text { Reducción } \\
\text { de gastos }\end{array}$} & Grupo 1 & 36 & 16,29 & 37 & 16,74 & 51 & 23,08 & 41 & 18,55 & 56 & 25,34 & \multirow[b]{3}{*}{0,1986} \\
\hline & & Grupo 2 & 54 & 11,87 & 73 & 16,04 & 119 & 26,15 & 74 & 16,26 & 135 & 29,67 & \\
\hline & & Grupo 3 & 4 & 19,05 & 7 & 33,33 & 3 & 14,29 & 1 & 4,76 & 6 & 28,57 & \\
\hline $\mathbf{v}$ & \multirow{3}{*}{$\begin{array}{c}\text { Optimizar } \\
\text { tiempo } \\
\text { estudio } \\
\end{array}$} & Grupo 1 & 47 & 21,27 & 58 & 26,24 & 53 & 23,98 & 27 & 12,22 & 36 & 16,29 & \multirow[b]{3}{*}{0,1181} \\
\hline $\mathbf{E}$ & & Grupo 2 & 79 & 17,36 & 94 & 20,66 & 117 & 25,71 & 69 & 15,16 & 96 & 21,1 & \\
\hline $\mathbf{N}$ & & Grupo 3 & 8 & 38,1 & 5 & 23,81 & 5 & 23,81 & 2 & 9,52 & 1 & 4,76 & \\
\hline $\mathbf{T}$ & \multirow{3}{*}{$\begin{array}{c}\text { Mayor } \\
\text { comodidad }\end{array}$} & Grupo 1 & 35 & 15,84 & 37 & 16,74 & 63 & 28,51 & 32 & 14,48 & 54 & 24,43 & \multirow[b]{3}{*}{0,5641} \\
\hline A & & Grupo 2 & 60 & 13,19 & 95 & 20,88 & 116 & 25,49 & 72 & 15,82 & 112 & 24,62 & \\
\hline $\mathbf{J}$ & & Grupo 3 & 4 & 19,05 & 3 & 14,29 & 9 & 42,86 & 2 & 9,52 & 3 & 14,29 & \\
\hline A & \multirow{3}{*}{$\begin{array}{l}\text { Evitar el } \\
\text { cadáver }\end{array}$} & Grupo 1 & 193 & 87,33 & 11 & 4,98 & 8 & 3,62 & 2 & 0,9 & 7 & 3,17 & \multirow[b]{3}{*}{0,5953} \\
\hline $\mathbf{S}$ & & Grupo 2 & 420 & 92,31 & 14 & 3,08 & 9 & 1,98 & 2 & 0,44 & 10 & 2,2 & \\
\hline & & Grupo 3 & 20 & 95,24 & 0 & 0 & 1 & 4,76 & 0 & 0 & 0 & 0 & \\
\hline & \multirow{3}{*}{$\begin{array}{c}\text { Flexibilidad } \\
\text { horaria }\end{array}$} & Grupo 1 & 50 & 22,62 & 52 & 23,53 & 57 & 25,79 & 28 & 12,67 & 34 & 15,38 & \multirow[b]{3}{*}{0,3902} \\
\hline & & Grupo 2 & 79 & 17,36 & 103 & 22,64 & 124 & 27,25 & 67 & 14,73 & 82 & 18,02 & \\
\hline & & Grupo 3 & 6 & 28,57 & 4 & 19,05 & 8 & 38,1 & 0 & 0 & 3 & 14,29 & \\
\hline & \multirow{3}{*}{$\begin{array}{c}\text { Estudio } \\
\text { a propio } \\
\text { ritmo } \\
\end{array}$} & Grupo 1 & 87 & 39,37 & 42 & 19 & 48 & 21,72 & 23 & 10,41 & 21 & 9,5 & \multirow[b]{3}{*}{0,1777} \\
\hline & & Grupo 2 & 180 & 39,56 & 100 & 21,98 & 107 & 23,52 & 31 & 6,81 & 37 & 8,13 & \\
\hline & & Grupo 3 & 14 & 66,67 & 3 & 14,29 & 4 & 19,05 & 0 & 0 & 0 & 0 & \\
\hline
\end{tabular}

Tabla 2- Valoración de las ventajas atribuidas a la virtualidad

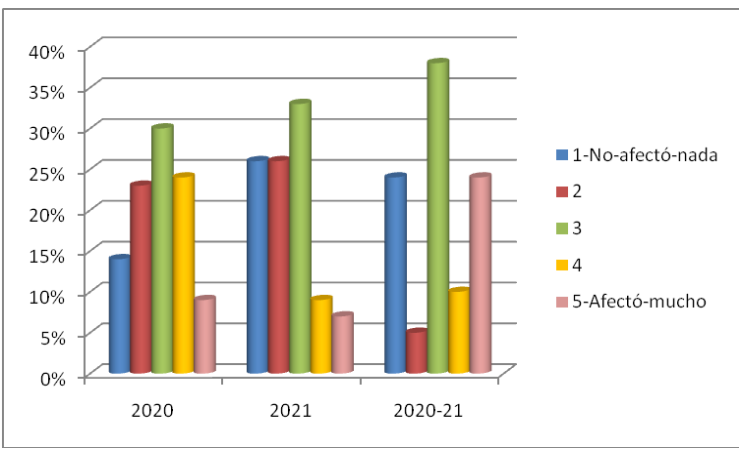

Gráfico 5- Desventaja de la duración de las actividades de docencia-aprendizaje.

Los factores que pudieron afectar el estudio virtual fueron evaluados del siguiente modo: A) Las distracciones familiares tuvieron incidencia importante $(39 \%, 33 \%$ y $57 \%$ respectivamente, para valores Likert 4 y 5) sin que alcanzara diferencia de significación entre el grupo I y II $(\mathrm{p}=0,1069)$ pero sí al relacionarlo con el grupo III $(p=0,0177)$.a quienes parece haberles afectado más que a los otros. Gráfico 4. B) La duración de las actividades virtuales afectó más al grupo I y III (33\%) y menos al grupo II (15\%) para los valores 4 y $5 \quad(p=<0,0001)$, sin alcanzar porcentajes muy altos. Los resultados están representados en el Gráfico 5. C) La falta de acceso al cadáver y preparados anatómicos afectó mucho a los estudiantes independientemente del grupo $(p=0,6876)$, con porcentajes de $86 \%$ (grupos I y II) y $90 \%$ (grupo III). D) La ansiedad también incidió en forma importante $(62 \%$ - 59\% - 67\% para valores 4 y 5$)$ y $\sin$ diferencia estadística entre los grupos $(p=0,1555)$. Gráfico 6. E) La falta de motivación fue un factor importante para todos los grupos 
pero con diferencias $(\mathrm{p}=0,0020)$. El máximo de afectación lo refieren los estudiantes del grupo III (81\%), seguidos por el grupo I (66\%) y por último el grupo II (53\%) para valores 4 y 5 . Gráfico 7 . F y G) La dificultad para concentrarse y la falta de contacto personal con los compañeros también alcanzaron valores Likert altos en todos los grupos y sin diferencias significativas internas ( $p=0,1906$ y $p=0,1092$ para cada item). Gráficos 8 y 9. (Ver Tabla 3$)$.

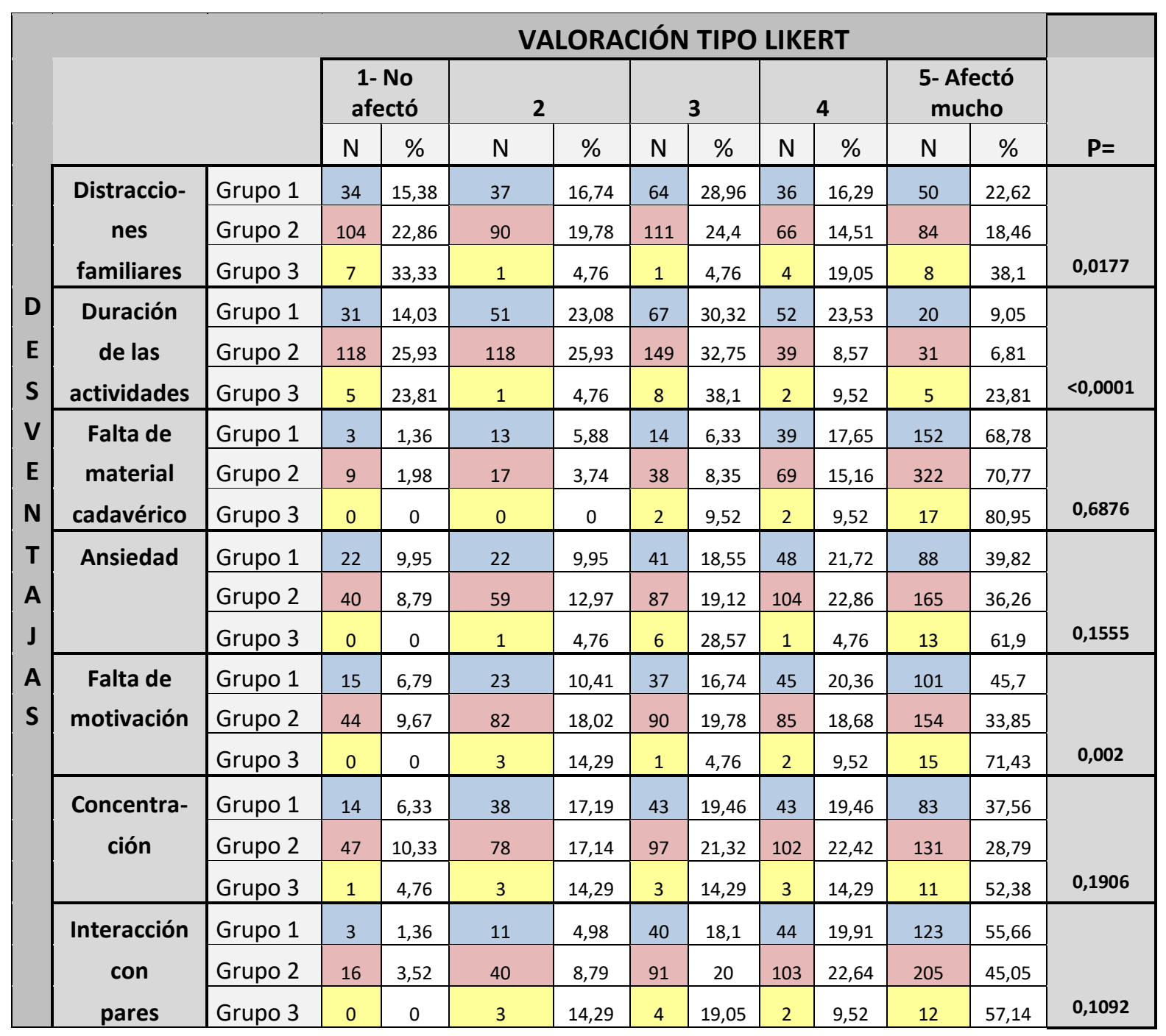

Tabla 3- Desventajas de la enseñanza-aprendizaje virtual

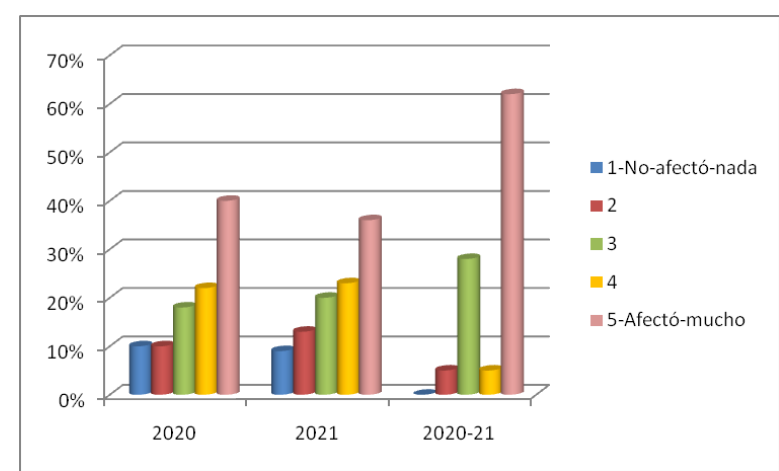

Gráfico 6- Incidencia de la ansiedad como desventaja para el estudio virtual

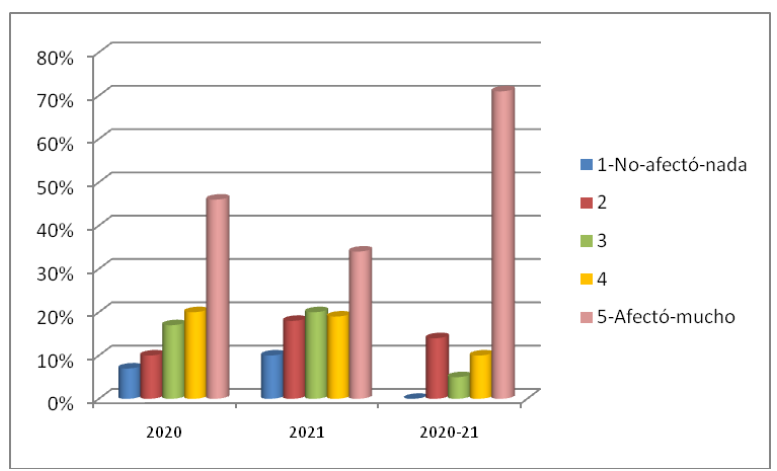

Gráfico 7- Incidencia de la falta de motivación en el estudio virtual 
La satisfacción que los encuestados manifestaron con las clases teóricas $(70 \%-75 \%-67 \%$ respectivamente para la valoración 3-4) alcanzó valores medio a alto, con diferencia significativa entre los tres grupos $(p=0,0064$ entre los grupos I y II, y $p=0,0406$ para la diferencia entre los 3 grupos). El grupo Il presentó valoración más alta.

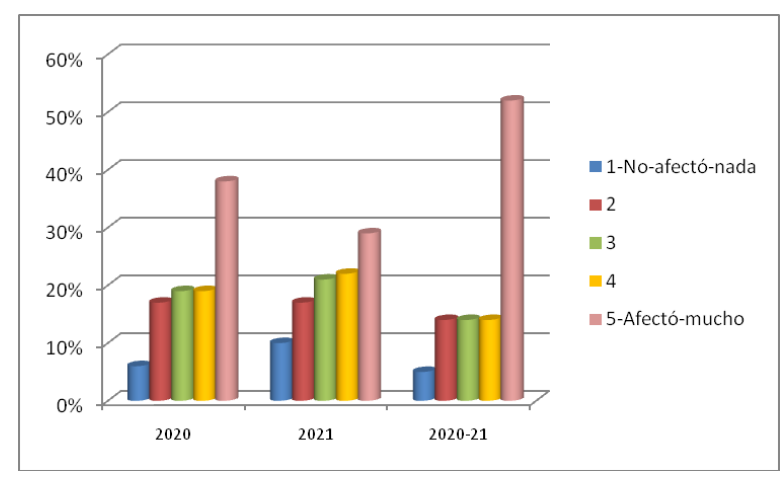

Gráfico 8- Representa la valoración de la dificultad para concentrarse.

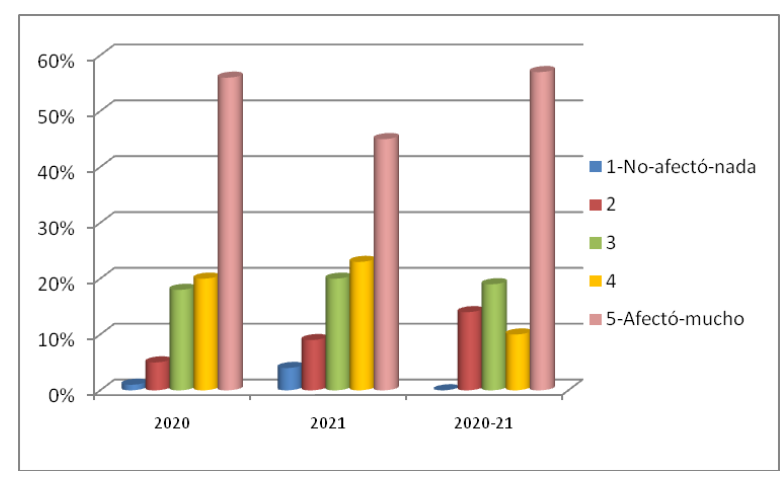

Gráfico 9- Incidencia de la falta de contacto con sus compañeros
Gráfico 10. Mientras que la satisfacción con los trabajos prácticos fue algo menor $(52 \%, 64 \%$ y $52 \%$ respectivamente para las calificaciones $3-4$ ) y mantuvo la diferencia a favor de valores más altos en el grupo II ( $p=0,0004)$. Gráfico 11. Los resultados específicos se encuentran en la Tabla 4.

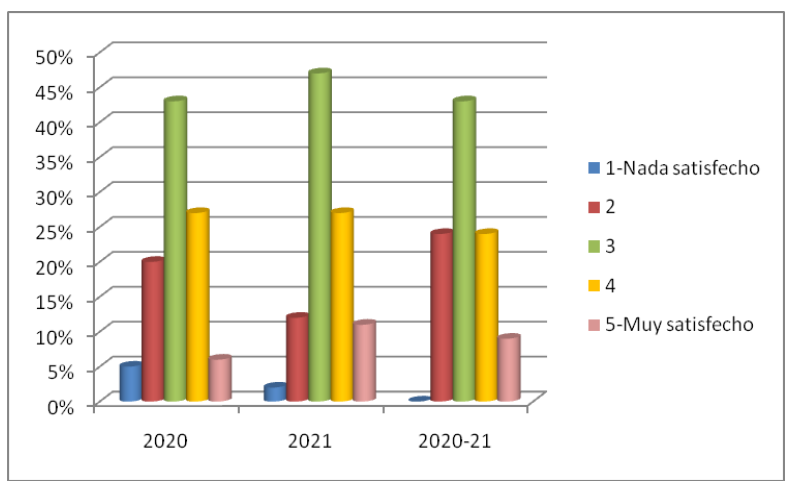

Gráfico 10- Satisfacción con las clases teóricas.

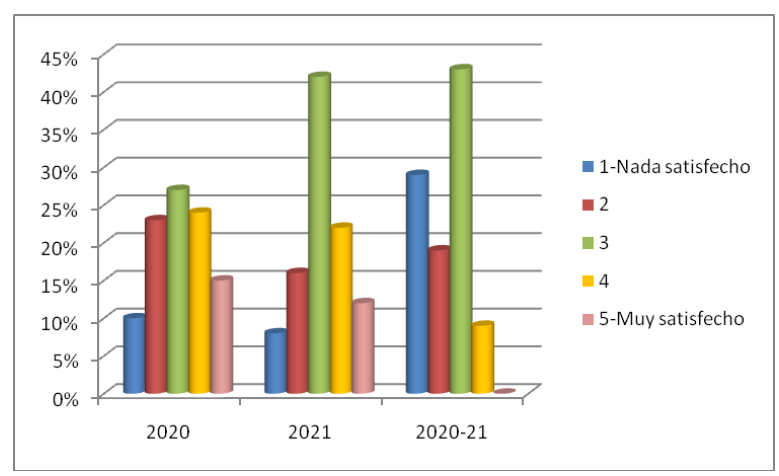

Gráfico 11- Satisfacción con los trabajos prácticos.

\begin{tabular}{|c|c|c|c|c|c|c|c|c|c|c|c|c|c|}
\hline \multirow{9}{*}{$\begin{array}{l}\text { SA } \\
\text { TIS } \\
\text { FAC } \\
\text { CIÓN }\end{array}$} & & & \multicolumn{10}{|c|}{ VALORACIÓN TIPO LIKERT } & \multirow[b]{3}{*}{$P=$} \\
\hline & & & \multicolumn{2}{|c|}{ 1- Nada } & \multicolumn{2}{|c|}{2} & \multicolumn{2}{|c|}{3} & \multicolumn{2}{|c|}{4} & \multicolumn{2}{|c|}{ 5-Mucho } & \\
\hline & & & $\mathrm{N}$ & $\%$ & $\mathrm{~N}$ & $\%$ & $\mathrm{~N}$ & $\%$ & $\mathrm{~N}$ & $\%$ & $\mathrm{~N}$ & $\%$ & \\
\hline & \multirow{3}{*}{$\begin{array}{c}\text { Clases } \\
\text { teóricas }\end{array}$} & Grupo 1 & 9 & 4,07 & 45 & 20,36 & 95 & 42,99 & 59 & 26,7 & 13 & 5,88 & \multirow[b]{3}{*}{0,0406} \\
\hline & & Grupo 2 & 11 & 2,42 & 53 & 11,65 & 216 & 47,47 & 124 & 27,25 & 51 & 11,21 & \\
\hline & & Grupo 3 & 0 & 0 & 5 & 23,81 & 9 & 42,86 & 5 & 23,81 & 2 & 9,52 & \\
\hline & \multirow{3}{*}{$\begin{array}{l}\text { Trabajos } \\
\text { prácticos }\end{array}$} & Grupo 1 & 23 & 10,41 & 51 & 23,08 & 60 & 27,15 & 54 & 24,43 & 33 & 14,93 & \multirow[b]{3}{*}{0,0004} \\
\hline & & Grupo 2 & 36 & 7,69 & 74 & 16,26 & 192 & 42,2 & 99 & 21,76 & 55 & 12,09 & \\
\hline & & Grupo 3 & 6 & 28,57 & 4 & 19,05 & 9 & 42,86 & 2 & 9,52 & 0 & 0 & \\
\hline
\end{tabular}

Tabla 4- Nivel de satisfacción de los alumnos con las clases teóricas y prácticas.

Permanecer en el domicilio, sin asistencia a las actividades habituales para el cursado de la asignatura tuvo una notable repercusión negativa en los estudiantes con valores 4-5 de afectación $(58 \%, 51 \%$ y $81 \%)$. No hubo diferencia entre los grupos I y || $(p=0,2525)$ pero se alcanzó la 
significación al incorporar la comparación con el grupo III ( $p=<0,0001)$, ya que este último refiere haber sido el mayormente afectado. Gráfico 12.

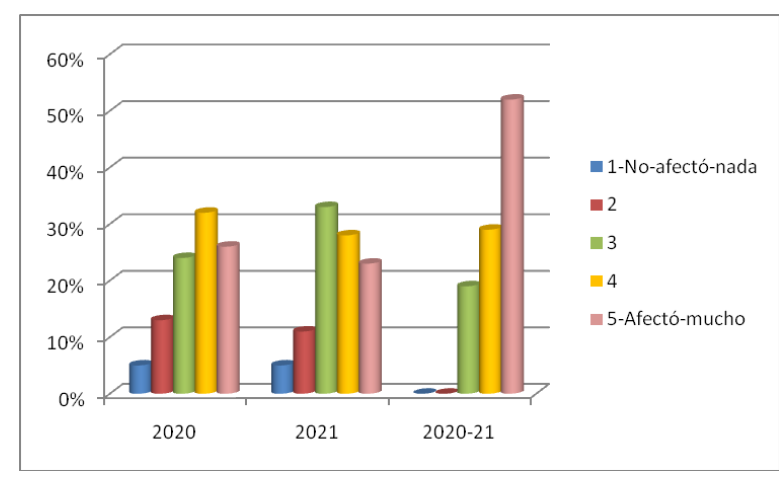

Gráfico 12- Grado de afectación para el estudio de la Anatomía generado por la permanencia en el domicilio, con carencia actividad presencial.

El tiempo destinado al estudio de la asignatura osciló entre 2 y 8 horas por día en todos los grupos. En los grupos I y II predominó la dedicación por 4 a 6 horas diarias, pero la comparación se vuelve significativa al considerar el grupo III que refiere haber mayoritariamente estudiado entre 2 y 4 horas diarias $(p=0,0295)$. Gráfico 13 El tiempo dedicado al estudio de preparados anatómicos fue significativamente mayor en el grupo I ( $p=0,0016)$ Gráfico 14.

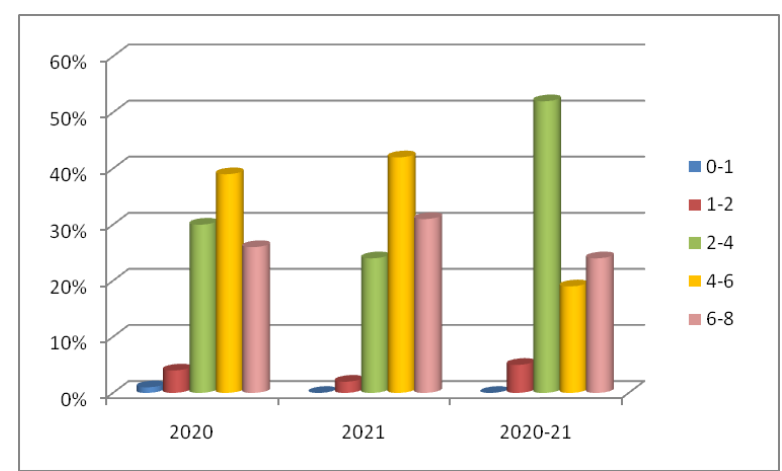

Gráfico 13- Tiempo destinado al estudio de la Anatomía en general.

El $94 \%, 96 \%$ y $100 \%$ de los alumnos consideraron que contar con cuerpos para el aprendizaje de la Anatomía es muy importante $(p=0,1862)$. Los restantes asignaron menor importancia, pero nadie lo consideró como "nada importante". $Y$ para el $100 \%$, el $99 \%$ y el $95 \%$ el conocimiento anatómico es muy importante para su carrera $(p=0,1758)$.

Los estudiantes evaluaron la incidencia de la modalidad de cursado durante la pandemia en su formación médica como altísima y sin diferencia estadísticamente significativa entre los grupos $(p=0,4322)$. Gráfico 15.

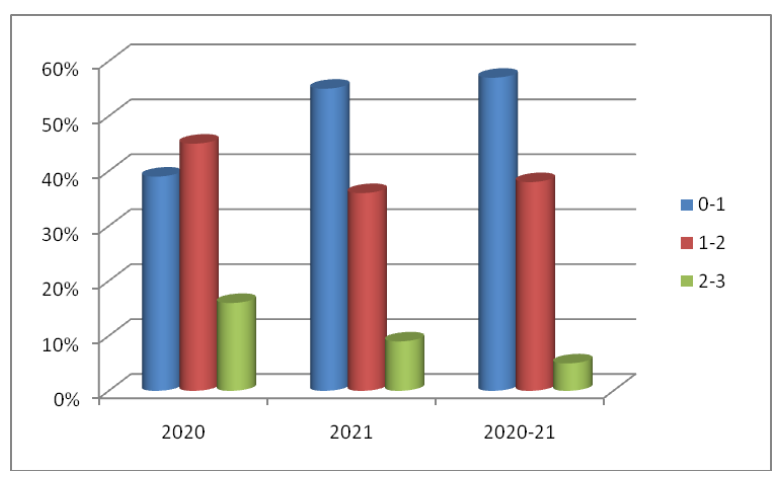

Gráfico 14- Tiempo dedicado al estudio de preparados anatómicos.

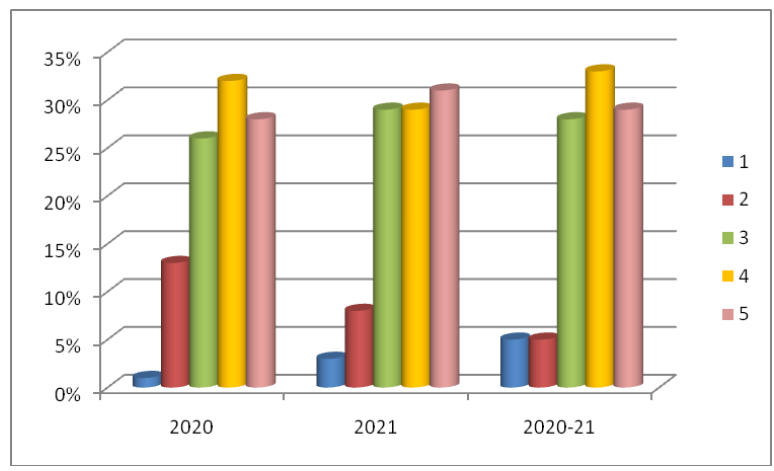

Gráfico 15- Incidencia de la modalidad de cursado durante la pandemia en su formación médica.

El cadáver es considerado como el primer paciente para un estudiante de Medicina. La opinión de los encuestados fue que "no es importante" (1,81\%-1,32\%-0\%), "espero recuperarlo este año con las actividades presenciales" (7\%-46\%-48\%), "podré recuperar esta experiencia porque soy ayudante" $(13,57 \%$, $0 \%, 0 \%)$, "es una pérdida irreparable" (7\%-5\%$0 \%$ ), "espero que la Facultad implemente algún modo de recuperación" (69\%-46\%-45\%), "otro" (1\%-2\%-5\%, aunque muchas consideraciones pudieron incluirse en los ítems anteriores).

Considerando a quienes no habían tenido experiencia previa con el estudio virtual, pudimos determinar que estos estudiantes solo manifestaron diferencia significativa con los demás en mayores problemas para el acceso a internet $(p=0,0382)$ y una menor ventaja de la virtualidad para optimizar el tiempo de estudio $(p=0,0485)$; aún así no refirieron que afectara el acceso a las actividades ni a las consultas $(p=0,0638$ y $p=0,5420)$. No hubo diferencia en el ahorro de tiempo ni en los gastos ( $p=3890$ y $p=0,2976)$, 
tampoco les afectó más la duración de las actividades $(p=0,2729)$ ni sufrieron mayor ansiedad ( $p=0,3647)$; y el tiempo destinado al estudio de la Anatomía en general y a las actividades prácticas no difirió por haber tenido menos experiencia en el estudio virtual $(p=0,2877$ y $p=0,2604$ respectivamente).

\section{DISCUSIÓN}

La mayoría de los artículos referidos a la educación médica y en especial anatómica, durante la pandemia COVID19, son artículos de opinión e informes sobre los recursos y estrategias aplicados para la enseñanza (Bond and Franchi, 2020; Murlimanju et al., 2020; Jones, 2021; Pawlina, 2021) o cuya publicación corresponde a los primeros meses de 2020 cuando la pandemia era aún incipiente. Gran parte de los estudios publicados difieren del nuestro por haber sido realizados en países del hemisferio norte y otros, que inician el ciclo lectivo en setiembre y habían completado gran parte del desarrollo de la Anatomía antes de instalarse la pandemia (Dost et al., 2020; Puljak et al., 2020; Banovac et al., 2021; Singal et al., 2021) cuando en las universidades argentinas el ciclo lectivo comienza en marzo y finaliza en octubre-noviembre de cada año. Así, solo algunos estudiantes argentinos habían iniciado las actividades docentes regulares antes de instalarse las restricciones y otros carecieron por completo de presencialidad en 2020.

Nuestro estudio está orientado a evaluar la percepción de los alumnos que cursaron y cursan actualmente en condiciones de restricción sobre la repercusión de la pandemia en la docencia-aprendizaje de la Anatomía.

Tal como refieren Dost et al. (2020), el estudio combinado mediante actividades presenciales y recursos digitales había probado su utilidad, pero tener que limitarse al aprendizaje virtual en Medicina y en Anatomía en especial, no tenía antecedentes y fue de complejo abordaje. Es sencillo comprender que la mayoría de los estudiantes que cursaron en 2020 no estuvieran familiarizados con el estudio virtual y que los porcentajes se elevaran en el grupo II, dada la experiencia del año anterior. Sin embargo, al comparar los resultados de Singal et al. (2021) con el grupo I (74\% - 26\%), por similitud temporal y de experiencias, la diferencia es estadísticamente significativa $(p=<0,0001)$ evidenciando una marcada deficiencia de nuestros estudiantes en el punto de partida de la virtualidad educativa. Los problemas de orden técnico no parecen haber sido de gran relevancia. La mayoría de los estudiantes no refieren haber tenido dificultades mayores para acceder a las actividades generadas por la Cátedra ni para realizar consultas en línea. La conexión a internet y los ruidos molestos tampoco adquirieron relevancia excepto para el grupo III. Solo el $20 \%$ del total refieren haber tenido falencias importantes en la provisión de internet, estableciendo una gran diferencia $(p=<0,0001)$ con el $80 \%$ en India (Singal et al., 2021).

La principal ventaja del estudio virtual parece haber sido el ahorro de tiempo, que se asemeja a los resultados de Dost et al. (2020); mientras que la reducción de gastos, el mejor aprovechamiento del tiempo de estudio, la comodidad y la flexibilidad en los horarios recibieron una valoración variable y con distribución similar entre los grupos. Por otro lado, una gran mayoría de los alumnos rechazaron que no enfrentar el contacto directo con los cadáveres y, en menor grado estudiar a su propio ritmo, fueran ventajas. Indudablemente, más allá de las dificultades que pudiera causar esa primera experiencia con los cuerpos, los alumnos valoraron como imprescindible la contribución al estudio que les ofrece el material cadavérico para la comprensión y percepción de realidad.

Las desventajas evaluadas por los alumnos fueron más frecuentes en el grupo I y III por lo que parecerían haber tenido mayor repercusión durante el año 2020 o bien los estudiantes que cursan en 2021 tienen mejores expectativas acerca de la reversión de las consecuencias por pandemia. La falta de acceso a los cuerpos y preparados anatómicos reales, la ansiedad, la falta de motivación para el estudio y de contacto con sus compañeros, y la dificultad para concentrarse fueron los principales factores adversos, mientras que las distracciones familiares, el internet y la duración de las actividades fueron considerados de incidencia media. Es complejo comparar con los estudiantes indios que disecan durante el curso de Anatomía, pero la importancia de disponer de cuerpos y material cadavérico alcanzó niveles relevantes en ambos casos, siendo el $98 \%$ asignado por los encuestados en nuestro estudio superior al $88 \%$ $(p=<0,0001)$ de Singal et al. (2021). La falta de acceso al laboratorio y la práctica en Anatomía también fue lo peor (73\%) de la enseñanza virtual para los estudiantes colombianos (SuárezEscudero et al., 2021)

El 58\% que manifestó valores 4-5 en falta de motivación para el estudio fue significativamente menor $(p=<0,0001)$ que el $69 \%$ de los alumnos indios. El $90 \%$ de los encuestados por Singal et al (2021) manifestó consecuencias negativas por la falta de interacción con sus compañeros, que 
se redujo a $70 \%$ en nuestro caso $(p=<0,0001)$, sin embargo, deberíamos considerar que los alumnos de nuestro estudio no establecieron una relación próxima en este sentido porque no tuvieron contacto previo a la instauración de las medidas restrictivas.

La satisfacción de los estudiantes respecto de las actividades docentes tuvo término medio, ligeramente mayor para las clases teóricas que para los trabajos prácticos; similar a las manifestaciones expresadas por los estudiantes de Medicina en el trabajo de Suárez-Escudero et al. (2021). Aún así, estos resultados fueron mejores $(p=<0,0001)$ que los expresados por los estudiantes indios (Singal et al., 2021) para quienes el $8,8 \%$ fue nada satisfactorio (vs $2,87 \%$ en este estudio) y otro $8,8 \%$ fue muy satisfactorio (vs $9,47 \%$ ).

La permanencia en el domicilio, con falta de asistencia a los lugares habituales para el aprendizaje fue evaluada como un daño importante tanto para los estudiantes indios como para nuestros encuestados. A pesar de ello, el nivel promedio de daño evaluado fue menor en nuestros grupos, con un $54 \%$ vs $68 \%$ reportado por Singal et al. $(p=<0,0001)$.

Que los alumnos del grupo II dedicaran similar tiempo que los del grupo I al estudio teórico pero menor al estudio de los preparados anatómicos puede estar asociado a la posibilidad de concurrir a la Cátedra para cumplir ese objetivo, aunque fuera en tiempo reducido. El tiempo global destinado al estudio fue similar al reportado por Banovac et al. (2021) para el periodo de estudio en línea. Sin embargo, la menor dedicación de los estudiantes del grupo III, tanto para el estudio teórico como para el práctico, podría estar relacionado con su situación particular (alumnos que no pudieron cumplimentar exitosamente el primer año en 2020).

Es de destacar que no evidenciamos diferencias significativas entre los alumnos de Córdoba y aquellos originarios de otras provincias 0 extranjeros.

La gran mayoría de los encuestados consideró fundamental disponer de cuerpos para el estudio de la Anatomía (en total acuerdo con lo expresado por Bond y Franchi, 2020) y el conocimiento anatómico para el desarrollo de la carrera. En su opinión, la pandemia tendrá una incidencia muy negativa en sus carreras, en un total acuerdo con las expresiones vertidas por Murlimanju et al. (2020). Con respecto a la posibilidad de recuperar la experiencia del "primer paciente", confían en que la Facultad implemente algún mecanismo de recuperación y/o que durante 2021 las actividades presenciales permitan acceder a ello. Si bien coincidimos con Pawlina (2021) en no descartar el valor de la pandemia para incentivar el desarrollo de las tecnologías digitales aplicadas al estudio de la Anatomía, insistimos en el valor del cuerpo humano como objeto final del estudio y trabajo del profesional médico (Jones, 2021). Con seguridad, la experiencia de la pandemia incrementará el espectro de recursos didácticos y reafirmará los ejes de la formación médica.

La principal limitación de este estudio está determinada por tratarse de una evaluación subjetiva sobre la percepción de los estudiantes y que no está vinculada a resultados académicos.

Como conclusión podemos considerar que si bien nuestros estudiantes iniciaron este proceso con un gran déficit de experiencia para el estudio virtual, durante su evolución parecen haber mejorado comparativamente con otras publicaciones. La falta de acceso al material cadavérico específico para el aprendizaje de la asignatura tuvo una gran repercusión negativa, aún disponiendo de otros recursos digitales, fotografías y videos de cuerpos reales. La carencia de contacto regular con sus compañeros y docentes, la ansiedad, falta de motivación y dificultad para concentrarse también los afectaron en forma significativa. Aparentemente la sumatoria de estas y otras dificultades pudieron ser causantes de fracasos en el curso, según manifiestan los alumnos recursantes. Se evidencia mayor expectativa en los alumnos 2021 para alcanzar mejoras en el proceso de aprendizaje debido a algunas flexibilidades en las medidas restrictivas. Este estudio no evalúa resultados académicos pero según la percepción de los estudiantes, las limitaciones impuestas por la pandemia están afectando severamente su formación, generando incertidumbre y poniendo en riesgo la formación de recursos humanos para la salud.

\section{Conflicto de Interés \\ Ninguno}

\section{Financiamiento \\ Ninguno}

\section{Aprobación Ética \\ No necesaria}

\section{Consentimiento Informado}

Los estudiantes fueron informados sobre la finalidad de la encuesta y que los resultados serían publicados. La participación en la encuesta fue anónima y voluntaria, y al completarla brindaron su conformidad. 


\section{Contribuciones}

SNB: administración del proyecto, análisis formal, conceptualización, escritura, revisión y edición, metodología y supervisión. LC: Investigación y borrador original. JPN: Investigación y borrador original. MPP: Investigación y borrador original. VSB: Investigación. MMV: Investigación. MAP: Investigación. NL: Investigación.

\section{AGRADECIMIENTOS}

Los autores agradecen a todos aquellos que voluntariamente donan sus cuerpos para que luego de fallecer contribuyan a la docencia e investigación universitaria.

\section{BIBLIOGRAFÍA}

Arraez-Aybar LA, Bueno-López JL, Moxham B. 2014. Anatomists' views on human body dissection and donation: An international survey. Annals of Anatomy 196: 376-86.

Banovac I, Katavic V, Blazevic A, Bicanic I, Hladnik A, Kovacic N, Petanjek Z. 2021. The anatomy lesson of the SARS-CoV-2 pandemic: Irreplaceable tradition (cadaver work) and new didactics of digital technology. Croat Med J. 62: 173-86

Bond G, Franchi T. 2020. Resuming cadaver dissection during a pandemic. Medical Education Online 26: 1842661. doi: 10.1080/10872981.2020.1842661

Azer SA, Eizenberg N. 2007. Do we need dissection in an integrated problem-based learning medical course? Perceptions of firstand second-year students. Surg Radiol Anat 29: $173-80$

Biasutto SN, Caussa LI, Criado del Río LE. 2006. Teaching Anatomy: Cadavers vs. Computers. Annals of Anatomy 188: $187-90$

Bond G, Franchi T. 2020. Resuming cadaver dissection during a pandemic. Medical Education Online 26: 1842661. doi: 10.1080/10872981.2020.1842661

Cahill KC, Ettarh RR. 2008. Student attitudes to whole body donation are influenced by dissection. Anat Sci Ed 1: 212-16.

Dereje G. 2014. Reaction of medical students to experiences in dissection room. Ethiopian $\mathrm{J}$ Health Sci 20: 337-42.

Deutschman MC, Hyson AR, Seithers LC, Jensen M. 2021. Teaching Interrupted: How COVID-19 Turned Thoughts into Practice. J Microbiol Biol Educ 22 doi: 10.1128/jmbe. v22i1.2613
Dost S, Hossain A, Shehab M, Abdelwahed A, Al-Nusair L. 2020. Perceptions of medical students towards online teaching during the COVID-19 pandemic: A national cross-sectional survey of 2721 UK medical students. BJM Open doi: 10.1136/bmjopen-2020-042378.

Jones DG. 2021. Anatomy in a Post-Covid-19 World: Tracing a New Trajectory. Anat Sci Educ 14: 148-53.

Longhurst GJ, Stone DM, Dulohery K, Scully D, Campbell T, Smith CF. 2020. Strength, Weakness, Opportunity, Threat (SWOT) Analysis of the Adaptations to Anatomical Education in the United Kingdom and Republic of Ireland in Response to the Covid-19 Pandemic. Anat Sci Educ 13: 298-308

Murlimanju BV, Agrawal A, Pai MM, Prabhu LV, Prameela MD, Moscote-Salazar LR. 2020. Reply to: Cadaverless anatomy: Darkness in the times of pandemic Covid-19. Morphologie 104: 297-98.

Narvaez-Hernandez E, Murillo-Rabago El. 2014. Herramientas de estudio utilizadas por los alumnos de ciencias de la salud en la materia de anatomía. Inv Ed Med 3: 204-08.

Pather N, Blyth P, Chapman JA, Dayal MR, Flack NAMS, Fogg $Q A$, Green RA, Hulme AK, Johnson IP, Meyer AJ, Morley JW, Shortland $P J$, Štrkalj G, 1 Štrkalj $M$, Valter $K$, Webb $A L$, Woodley SJ, Lazarus MD. 2020. Forced Disruption of Anatomy Education in Australia and New Zealand: An Acute Response to the Covid-19 Pandemic. Anat Sci Educ 13: 284-97.

Pawlina W. 2021. Effects of Covid-19: The need to assess the real value of Anatomy education. Anat Sci Educ 14:129-31.

Puljak L, Civljak M, Harmina A, Malisa S, Cavic $D$, Klinec C, Aranza D, Mesaric J, Skitarelic N, Zoranic S, Majstorovic D, Neuberg M, Miksic S, Ivanisevic K. 2020. Attitudes and concerns of undergraduate university health sciences students in Croatia regarding complete switch to e- learning during COVID-19 pandemic: a survey. BMC Medical Education 20: 416-26.

Shahrvini B, Baxter S, Coffey CS, MacDonald BV, Lande L. 2021. Pre-clinical remote undergraduate medical education during the COVID-19 pandemic: A survey study. BMC Medical Education. doi: 10.21203/rs.3.rs33870/v1.

Singal A, Bansal A, Chaudhary $P$, Singh $H$, Patra A. 2021. Anatomy education of medical and dental students during COVID-19 pandemic: a reality check. Surg Radiol Anat 43: 515-21.

Singal A, Bansal A, Chaudhary P. 2020. Cadaverless anatomy: Darkness in the times of pandemic Covid-19. Morphologie 104: 147-50. 
Suárez-Escudero JC, Bedoya Muñoz LJ, Posada Jurado MC, Arboleda Carmona EP, Urbina Sánchez AJ, Ramírez Burgos S, Bohórquez Gutiérrez CA, Ferreira Morales JL. 2021. Percepción de los estudiantes sobre adaptaciones virtuales en cursos de anatomía humana por la contingencia SARS-CoV-2. Academia y Virtualidad 14: 151-68.

Sugand K, Abrahams P, Khurana A. 2010. The anatomy of anatomy: a review for its modernization. Anat Sci Educ 3: 83-93. 